\title{
Adding Temporal Semantics to Association Rules
}

\author{
Chris P. Rainsford ${ }^{1}$ and John F. Roddick ${ }^{2}$ \\ ${ }^{1}$ Defence Science and Technology Organisation, DSTO C3 Research Centre \\ Fernhill Park, Canberra, 2600, Australia. \\ chris.rainsford@dsto.defence.gov.au \\ ${ }^{2}$ Advanced Computing Research Centre, School of Computer and Information Science \\ University of South Australia, The Levels, Adelaide, 5095, Australia. \\ roddick@cis.unisa.edu.au
}

\begin{abstract}
The development of systems for knowledge discovery in databases, including the use of association rules, has become a major research issue in recent years. Although initially motivated by the desire to analyse large retail transaction databases, the general utility of association rules makes them applicable to a wide range of different learning tasks. However, association rules do not accommodate the temporal relationships that may be intrinsically important within some application domains. In this paper, we present an extension to association rules to accommodate temporal semantics. By finding associated items first and then looking for temporal relationships between them, it is possible to incorporate potentially valuable temporal semantics. Our approach to temporal reasoning accommodates both point-based and intervalbased models of time simultaneously. In addition, the use of a generalized taxonomy of temporal relationships supports the generalization of temporal relationships and their specification at different levels of abstraction. This approach also facilitates the possibility of reasoning with incomplete or missing information.
\end{abstract}

\section{Introduction}

Association rules have been widely investigated within the field of knowledge discovery q.v. ([1],[2],[7],[8],[9],[12],[14]). In this paper we present an extension to association rules to allow them to exploit the semantics associated with temporal data and particularly temporal interval data. Some work in the discovery of common sequences of events has been conducted q.v. ([3],[10],[15]). However, these algorithms are aimed at finding commonly occurring sequences rather than associations. Moreover, the algorithms only accommodate point-based events and this restricts both the potential semantics of knowledge that may be discovered and the data that can be learnt from. Other investigations have examined the discovery of association rules from temporal data, such that each discovered rule is weakened by a temporal dependency q.v. [5]. Özden et al. extend this to cyclic domains to describe associations that are strong during particular parts of a specified cycle [11]. This may be used to describe the behavior of rules that only hold true in summer or winter or during some other part of a given cycle. 
Conventional association rules do not accommodate the temporal relationships that may be intrinsically important in some application domains. Importantly, each basket of items is treated individually with no record of the associated customer or client who purchased these goods. However, where client histories exist, temporal patterns may be associated with their purchasing behaviour over time. Therefore it would useful to provide organisational decision-makers with this temporal information. Existing association rule algorithms do not support such temporal semantics. This paper addresses this issue by presenting an extension to association rules that accommodates both point-based and interval-based models of time simultaneously. In addition, the use of a generalised taxonomy of relationships supports the generalisation of temporal relationships and specification at different levels of abstraction. This approach also facilitates the possibility of reasoning with incomplete or missing information. This flexibility makes the proposed approach applicable to a wide array of application domains.

Although initially motivated by the desire to analyse large retail transaction databases, the general utility of association rules makes them applicable to a wide range of different learning tasks. For consistency the entities with which a history of transactions is associated are described here as clients. Clients may be associated with non-temporal properties such as sex, specific events such as item purchases or with attributes manifested over intervals such as bank balances, outstanding debts or insurance classifications. These properties are described as items and the set of items associated with a client is said to be their basket of items.

Association rules may be able tell us that Investment_ $\mathrm{X}$ is associated with Insurance_Y. Temporal associations may then tell us that Investment_X usually occurs after the start of Insurance_Y. This may indicate that customers start with an insurance policy and this becomes a gateway for other services such as Investment_X. A campaign marketing Investment_X to holders of Insurance $\bar{Y}$ may then be suggested.

In the next section temporal association rules are formally defined. Section 3 then discusses the temporal logic that underlies the proposed approach to learning temporal association rules. An overview of the learning algorithm is provided in Section 4. In Section 5 a summary of this paper and discussion of future research is provided.

\section{Temporal Association Rules}

A temporal association rule can be considered a conventional association rule that includes a conjunction of one or more temporal relationships between items in the antecedent or consequent. Building upon the original formalism in [1] temporal association rules can be defined as follows: Let $\mathrm{I}=\mathrm{I}_{1}, \mathrm{I}_{2}, \ldots, \mathrm{I}_{\mathrm{m}}$ be a set of binary attributes or items and $\mathrm{T}$ be a database of tuples. Association rules were first proposed for use within transaction databases, where each transaction $t$ is recorded with a corresponding tuple. Hence attributes represented items and were limited to a binary domain where $\mathrm{t}(\mathrm{k})=1$ indicated that the item $\mathrm{I}_{\mathrm{K}}$ had been purchased as part of the transaction, and $\mathrm{t}(\mathrm{k})=0$ indicated that it had not. However in a more general context t may be any tuple with binary domain attributes, which need not represent a transaction buy may simply represent the presence of some attribute value or range of 
values. Temporal attributes are defined as attributes with associated temporal points or intervals that record the time for which the item or attribute was valid in the modelled domain. Let $\mathrm{X}$ be a set of some attributes in I. It can be said that a transaction $t$ satisfies $X$ if for all attributes $I_{k}$ in $X, t(k)=1$. Consider a conjunction of binary temporal predicates $\mathrm{P}_{1} \wedge \mathrm{P}_{2} \ldots \wedge \mathrm{P}_{\mathrm{n}}$ defined on attributes contained in either $\mathrm{X}$ or $\mathrm{Y}$ where $\mathrm{n} \geq 0$. Then by a temporal association rule, we mean an implication of the form $\mathrm{X} \Rightarrow \mathrm{Y} \wedge \mathrm{P}_{1} \wedge \mathrm{P}_{2} \ldots \wedge \mathrm{P}_{\mathrm{n}}$, where $\mathrm{X}$, the antecedent, is a set of attributes in $\mathrm{I}$ and $\mathrm{Y}$, the consequent, is a set of attributes in $\mathrm{I}$ that is not present in $\mathrm{X}$. The rule $\mathrm{X} \Rightarrow \mathrm{Y} \wedge$ $\mathrm{P}_{1} \wedge \mathrm{P}_{2} \ldots \wedge \mathrm{P}_{\mathrm{n}}$ is satisfied in the set of transactions $\mathrm{T}$ with the confidence factor $0 \leq \mathrm{c}$ $\leq 1$ iff at least c\% of transactions in $\mathrm{T}$ that satisfy $\mathrm{X}$ also satisfy $\mathrm{Y}$. Likewise each predicate $\mathrm{P}_{\mathrm{i}}$ is satisfied with a temporal confidence factor of $0 \leq \mathrm{tc}_{\mathrm{Pi}} \leq 1$ iff at least $\mathrm{tc} \%$ of Transactions in $\mathrm{T}$ that satisfy $\mathrm{X}$ and $\mathrm{Y}$ also satisfy $\mathrm{P}_{\mathrm{i}}$. The notation $\mathrm{X} \Rightarrow \mathrm{Y} \mid \mathrm{c} \wedge$ $\mathrm{P}_{1}$ Itc $\wedge \mathrm{P}_{2}$ Itc $\ldots \wedge \mathrm{P}_{\mathrm{n}}$ Itc is adopted to specify that the rule $\mathrm{X} \Rightarrow \mathrm{Y} \wedge \mathrm{P}_{1} \wedge \mathrm{P}_{2} \ldots \wedge \mathrm{P}_{\mathrm{n}}$ has a confidence factor of $\mathrm{c}$ and temporal confidence factor of tc. As an illustration consider the following simple example rule:

policyC $\Rightarrow$ investA, productB $\mid 0.87 \wedge$ during(investA, policyC) 0.79 $\wedge$ before(productB, investA) | 0.91

This rule can be read as follows:

The purchase of investment $A$ and product $B$ are associated with insurance policy $C$ with a confidence factor of 0.87 . The investment in A occurs during the period of policy $C$ with a temporal confidence factor of 0.79 and the purchase of product $B$ occurs before investment $A$ with a temporal confidence factor of 0.91

Binary temporal predicates are defined using Allen's thirteen interval based relations and Freksa's neighbourhood relations and this will be discussed in the next section.

\section{Temporal Logic}

The expressiveness of temporal association rules is determined by the set of temporal predicates available to describe relationships between items. For our work, Allen's taxonomy of temporal relationships is adopted to describe the basic relationships between intervals [4]. These relationships become the basis for binary temporal predicates. Using these relations we are able to treat points as a special case of intervals where begin and end points are equal. To add extra expressive capability, Freksa's generalised relationships have also been adopted [6]. Freksa's neighbourhood relations generalise over Allen's relations and this allows the proposed algorithm to describe temporal relationships at multiple levels. Therefore several commonly occurring relationships can be summarised into single strong relationships. Both of these taxonomies are depicted in Figure 1. 


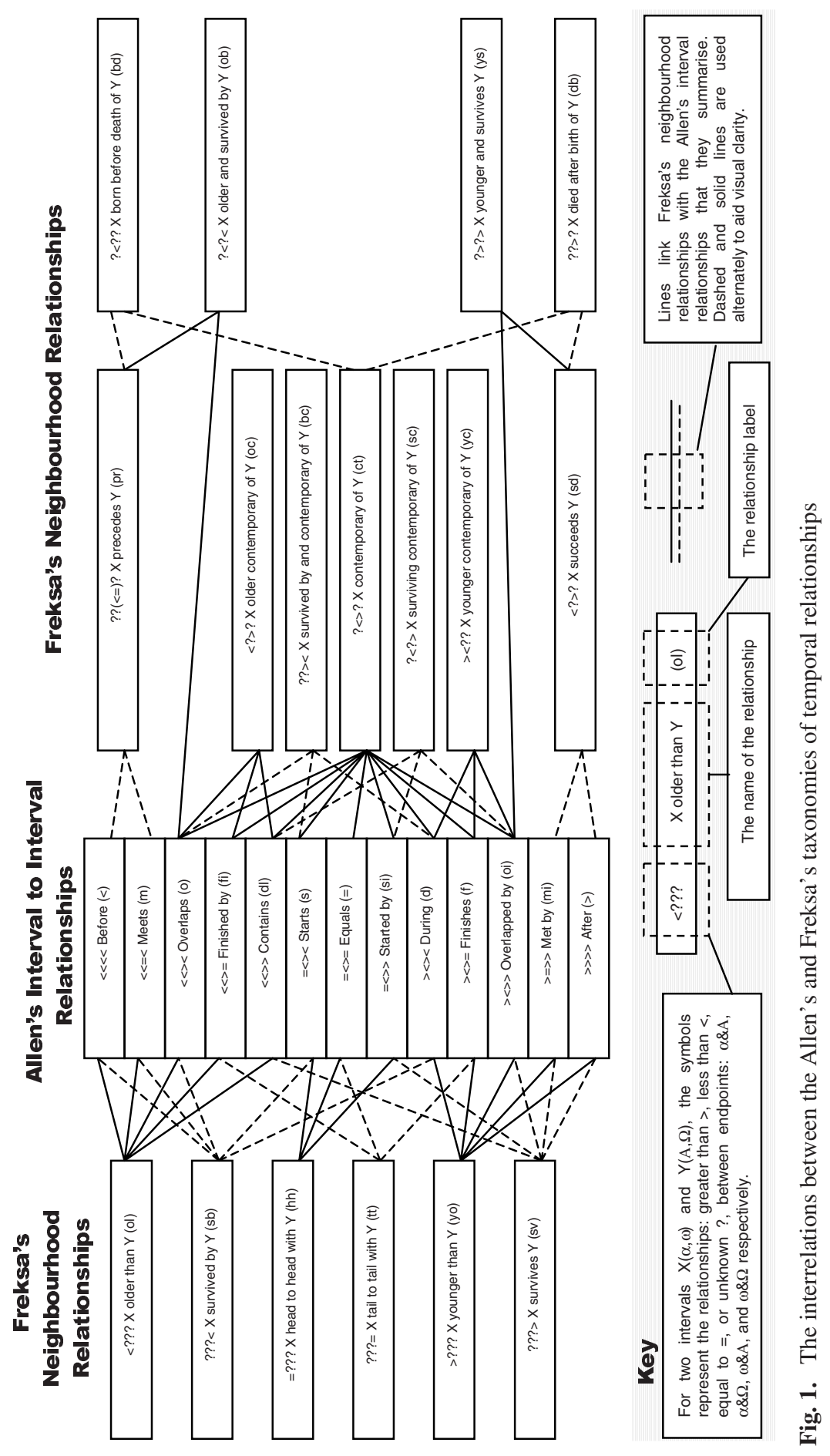




\section{The Learning Algorithm}

The temporal association learning algorithm can be seen as a four-stage process as depicted in Figure 2. A detailed description of this process is provided in [13]. The first stage of the learning process is the use of an association rule learning algorithm to generate an initial set of association rules. This process is independent of any specific association rule learning algorithm and this has the advantage of allowing the user to utilise the algorithm of their choice to learn the association rules. During this phase the temporal attributes associated with the items are ignored. The separation between the first and second phase allows the user to prune out uninteresting associations before proceeding with a temporal analysis. In the second phase all of the possible pairings of temporal items in each rule in the set of discovered association rules are generated. These pairing can then be tested to see if any strong temporal relationships exist between them.

In the third phase, the database is scanned to determine the temporal nature of the relationships between the candidate item pairings. Each tuple is checked to see if it supports a given rule. If support exists, then the temporal relationships between the items in that instance are recorded as one of Allen's thirteen basic relationships. The fourth phase of the learning algorithm is the derivation of temporal association rules based upon the original ruleset and the aggregation of temporal relationships found to exist between items. During this phase Allen's relationships may be generalised to Freksa's more general relationships so that the temporal confidence threshold can be met. Candidate relationships that do not meet the confidence threshold are discarded.

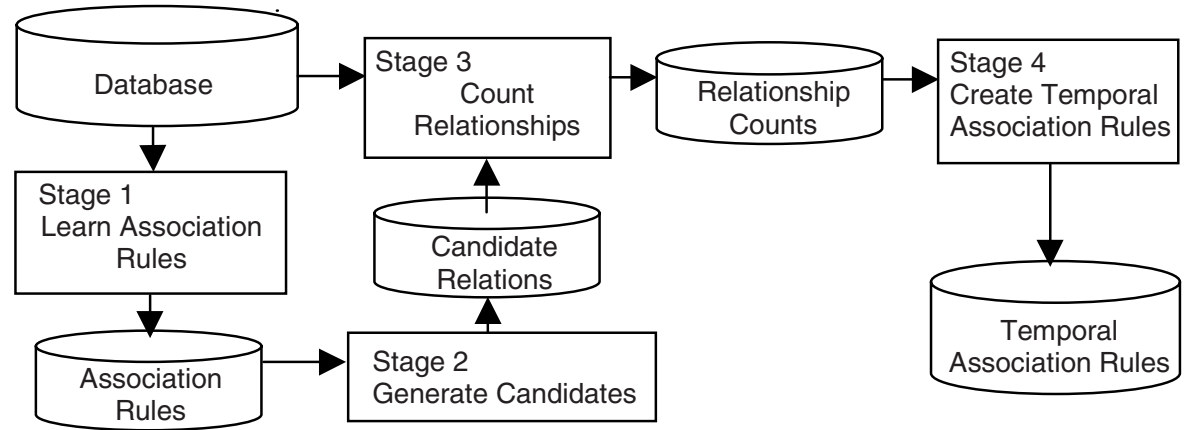

Fig. 2. The four-stage temporal association rule learning process.

\section{Summary}

In this paper we have presented an extension to association rules to accommodate both interval and point-based temporal semantics. This technique operates upon the output of existing association rule algorithms and therefore is capable of exploiting existing knowledge discovery resources. Our preliminary experiments have indicated that the performance of this algorithm is of a similar order to a comparatively implemented conventional association rule learning process q.v. [13]. The possibility 
of further experimentation and improvements to this algorithm includes reimplementation with enhancements to increase performance as well as application to real-world problem domains. An interface to facilitate the discovery and presentation of temporal associations is currently being developed.

\section{References}

1. Agrawal, A., Imielinski, T., Swami, A.: Mining Association Rules between Sets of Items in Large Databases. International Conference on Management of Data (SIGMOD'93), May (1993) 207-216.

2. Agrawal, A., Srikant, R.: (1994). Fast Algorithms for Mining Association Rules. 20 ${ }^{\text {th }}$ VLDB conference, September, Santiago, Chile. (1994) 487-499.

3. Agrawal, R., Srikant, R.: Mining Sequential Patterns. International Conference on Data Engineering (ICDE), March, Taipei, Taiwan (1995).

4. Allen, J. F.: Maintaining knowledge about temporal intervals. Communications of the ACM Vol 26. No.11 (1983).

5. Chen, X., Petrounias, I., Heathfield, H.: Discovering Temporal Association Rules in Temporal Databases. International Workshop on Issues and Applications of Database Technology (IADT98), July, Berlin, Germany.(1998) 312-319.

6. Freksa, C.: Temporal reasoning based on semi-intervals. Artificial Intelligence 54, (1992) 199-227.

7. Han, J., Fu, Y.: Discovery of Multiple-Level Association Rules from Large Databases, Technical Report, Simon Fraser University. (1995).

8. Koperski, K., Han, J.: Discovery of Spatial Association Rules in Geographic Information Databases. The $4^{\text {th }}$ International Synopsum on Large Spatial Databases, August, Maine. (1995) 47-66.

9. Mannila, H., Toivonen, H., Verkamo, A.I.: Efficient Algorithms for Discovering Association Rules. AAAI Workshop on Knowledge Discovery in Databases, July, Seattle, washington, U. Fayyad, M. and R. Uthurusamy (Eds) (1994) 181-192.

10. Mannila, H., Toivonen, H., Verkamo, A.I.: Discovery of frequent episodes in event sequences. Report C-1997-15, University of Helsinki, Department of Computer Science, February (1997).

11. Özden, B., Ramaswamy, S., Silberschatz, A.: Cyclic Association Rules, International Conference on Data Engineering, April, (1998).

12. Park, J.S., Chen, M., Yu, P.S.: An Effective Hash-Based Algorithm for Mining Association Rules. ACM SIGMOD (1995).

13. Rainsford, C.P., Accommodating Temporal Semantics in Knowledge Discovery and Data Mining, PhD Thesis, University of South Australia, (submitted)(1998).

14. Srikant, R., Agrawal, R.: Mining Generalized Association Rules. The $21^{\text {st }}$ International Conference on Very Large Databases, September, Zurich, Switzerland. (1995).

15. Srikant, R., Agrawal, R.: Mining Sequential Patterns: Generalizations and Performance Improvements. Fifth International Conference on Extending Database Technology (EDBT), March, Avignon, France.(1996). 